Check for updates

Cite this: J. Mater. Chem. C, 2018, 6, 10569

Received 26th April 2018, Accepted 4th September 2018 DOI: $10.1039 / c 8 t c 01998 c$

rsc.li/materials-c

\title{
Temperature dependence of the photo- and electroluminescence of poly( $p$-phenylene vinylene) based polymers $\dagger$
}

\author{
Irina Rörich, ${ }^{\text {ab }}$ Ann-Kathrin Schönbein, ${ }^{a}$ Deepthi Kamath Mangalore, ${ }^{a}$ \\ Anielen Halda Ribeiro, ${ }^{a}$ Christian Kasparek, ${ }^{a}$ Christian Bauer, $^{a}$ N. Irina Crăciun, ${ }^{a}$ \\ Paul W. M. Blom ${ }^{a}$ and Charusheela Ramanan 1 *a
}

\begin{abstract}
We show that the exciton transport and decay processes in two poly(p-phenylene vinylene) (PPV) based semiconducting polymers exhibit distinct temperature dependence based on the energetic disorder of the polymer. Time-resolved photoluminescence (TRPL) spectroscopy and Monte Carlo exciton diffusion simulations are used to disentangle the contributions from radiative and non-radiative decay processes, with the latter including non-radiative decay due to exciton diffusion toward trap sites. In a highly disordered polymer, the exciton lifetime and quantum yield are nearly temperature independent. In the case of a less disordered polymer, the exciton lifetime and quantum yield increase with decreasing temperature, due to both freezing out vibrations and less exciton quenching by slowing down the diffusion toward trap sites. We further demonstrate that the temperature dependence of the electroluminescence of polymer light-emitting diodes comprising these polymers is directly correlated with the photoluminescence behavior.
\end{abstract}

\section{Introduction}

Conjugated polymer materials for thin film optoelectronics are subject to energetic disorder arising from both molecular conformational irregularities as well as the sensitivity of these amorphous systems to film processing conditions. As a result, these organic semiconductors are commonly associated with strongly dispersive charge carrier mobilities and exciton diffusion characteristics as compared to their inorganic counterparts. ${ }^{1-3}$ In order to take advantage of the attractive properties of these systems, such as low-cost manufacturing and mechanical flexibility, it is essential to understand the interplay between energetic disorder and processes affecting optoelectronic performance. Herein, we characterized the effects of energetic disorder on photo and electroluminescence behavior in two poly( $p$-phenylene vinylene) (PPV) based semiconducting polymers used in polymer light-emitting diodes (PLEDs).

The device operation of a PLED is determined by three processes: charge carrier (electron and hole) injection from the electrodes, charge transport through the polymer active layer, and charge recombination generating an exciton, which

\footnotetext{
${ }^{a}$ Max Planck Institute for Polymer Research, Ackermannweg 10, 55128 Mainz, Germany.E-mail: ramanan@mpip-mainz.mpg.de

${ }^{b}$ Dutch Polymer Institute, P.O. Box 902, 5600 AX Eindhoven, The Netherlands

$\dagger$ Electronic supplementary information (ESI) available. See DOI: 10.1039/c8tc01998c
}

can then decay radiatively. ${ }^{4-6}$ Charge carrier and exciton transport in organic semiconductors can be regarded as a hopping process between localized states on conjugated chain segments that are inhomogeneously distributed in size and energy within a Gaussian density of states (DOS). ${ }^{1,7-9}$ The width of the Gaussian DOS $(\sigma)$ represents the degree of energetic disorder in the system. The three processes that drive PLED operation depend on the DOS: charge injection is mediated by the relative energy level matching to the electrodes, and charge transport and successive exciton diffusion, prior to radiative decay, depends on the energy level distribution. An exciton introduced into an arbitrary energy level of the DOS will subsequently migrate toward the lower-energy tail via dispersive relaxation steps. At quasi-equilibrium, the exciton reaches the energy level of the highest population of states, located at $-\sigma^{2} / k T$, where the hopping is determined by thermally-activated transfer. ${ }^{1,9}$ Thus, the energetic disorder resulting from molecular conformation, structural defects, and the variety of inter- and intrachain interactions due to the irregular morphologies, will govern the exciton diffusion and decay characteristics in PPV films for PLEDs. ${ }^{10,11}$ Time-resolved photoluminescence (PL) experiments enable characterization of the exciton diffusion behavior in conjugated polymers. An exciton, formed by either photo-excitation or Langevin recombination in a PLED, will decay back to the ground state within a lifetime $\tau_{\mathrm{f}}$. The PL quantum yield $\left(\phi_{\mathrm{f}}\right)$ represents the ratio between radiative and non-radiative processes. ${ }^{12}$ 


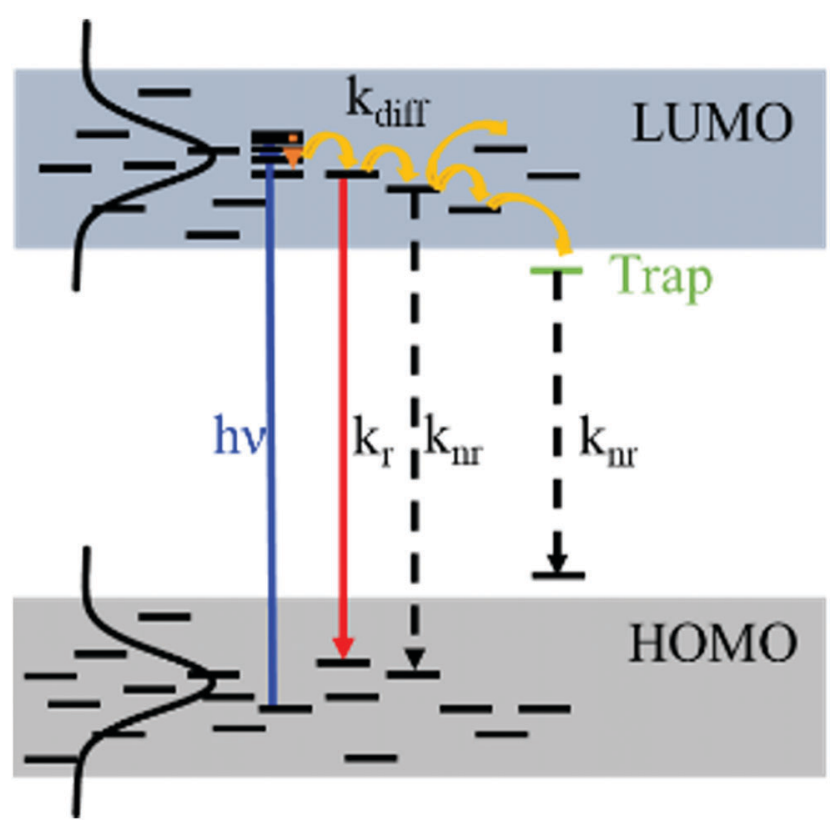

Fig. 1 The photophysical processes of excitons in a conjugated polymer thin film, which determine the exciton lifetime $\tau_{f}$ and the photoluminescence quantum yield, $\phi_{\mathrm{f}}$.

In a thin film with significant chromophore-chromophore interactions, energy-transfer will occur via hopping along interand intrachain segments until the exciton decays either radiatively or non-radiatively. Thus, the PL lifetime $\tau_{\mathrm{f}}$ and quantum yield $\phi_{\mathrm{f}}$ are governed by the intrinsic radiative $\left(k_{\mathrm{r}}\right)$ and nonradiative $\left(k_{\mathrm{nr}}\right)$ decay rates, as well as the exciton diffusion with a rate $k_{\text {diff }}$, which represents the non-radiative decay related to exciton quenching at defect sites (Fig. 1, eqn (1) and (2)). ${ }^{13}$

$$
\begin{aligned}
\tau_{\mathrm{f}} & =\frac{1}{\left(k_{\mathrm{r}}+k_{\mathrm{nr}}+k_{\mathrm{diff}}\right)} \\
\phi_{\mathrm{f}} & =\frac{k_{\mathrm{r}}}{\left(k_{\mathrm{r}}+k_{\mathrm{nr}}+k_{\mathrm{diff}}\right)}
\end{aligned}
$$

Previous work demonstrates that $\tau_{\mathrm{f}}$ and $\phi_{\mathrm{f}}$ directly correlate with the energetic disorder of conjugated polymers, where increased disorder slows down the diffusion of the exciton toward non-radiative quenching sites and strongly enhances both $\tau_{\mathrm{f}}$ and $\phi_{\mathrm{f}} \cdot{ }^{14}$ This is consistent with diffusion-limited exciton quenching at defects in organic semiconductors. ${ }^{15}$ It should be noted that while the electronic DOS relevant for charge transport and exciton DOS are not the same, both represent the energy level distribution in the polymer. Consistent with this and our previous results, Markov et al. observed a three orders of magnitude enhanced charge carrier mobility in PPV derivatives with decreasing energetic disorder. ${ }^{16}$

Although charge transfer and exciton diffusion processes are well studied in disordered conjugated polymers, a significant open question remains as to how the PL characteristics of a polymer influence the PLED conversion efficiency. For example, the PLED efficiency is charge carrier mobility independent, and is therefore expected to be temperature independent with regards to the active layer material. ${ }^{17,18}$ However, the PL behavior of a polymer thin film may vary with temperature as the chromophore-chromophore interactions will affect the balance of $k_{\mathrm{r}}, k_{\mathrm{nr}}$, and $k_{\mathrm{diff}}$. Since the PLED efficiency is governed by the formation of excitons, it follows that the respective temperature dependencies of the PLED and PL characteristics should have a direct correlation.

Here, we investigate this relationship by measuring the temperature-dependence of the PL and PLED efficiencies in the PPV derivatives BEH-PPV (poly[2,5-bis(2'-ethylhexyloxy)-1,4phenylene vinylene]) and SY-PPV (SuperYellow copolymer, Merck) (see Fig. 2 for chemical structures). These materials exhibit different degrees of energetic disorder, as the width of the charge carrier Gaussian DOS ( $\left.\sigma_{\text {electrical }}\right)$ is determined to be 0.092 and $0.140 \mathrm{eV}$ for BEH-PPV and SY-PPV, respectively. ${ }^{16}$ Furthermore, the exciton lifetime is strongly enhanced with increasing disorder, ranging from $180 \mathrm{ps}$ for BEH-PPV to $1.9 \mathrm{~ns}$ for SY-PPV. ${ }^{14}$ In the new work presented here, time-resolved photoluminescence spectroscopy (TRPL) at varying temperature shows a correlation between the conformational energetic disorder and the balance between radiative $\left(k_{\mathrm{r}}\right)$ and non-radiative decay processes $\left(k_{\mathrm{nr}}\right.$ and $k_{\text {diff)}}$. The thin film of SY-PPV, a highly disordered polymer, exhibits a nearly temperature independent photoluminescence quantum yield, while the more ordered BEH-PPV demonstrates a significant decrease in non-radiative quenching with decreasing temperature (decrease of $k_{\text {diff }}$ and $k_{\mathrm{nr}}$ ). We then fabricated PLEDs with these materials in order to correlate device behavior with the results from our optical experiments. We found that PLEDs based on the disordered SY-PPV show temperature independent current efficiencies, whereas the better ordered BEH-PPV demonstrates enhanced PLED efficiency with lowering temperature, consistent with the temperature dependence of the photoluminescence quantum yield. In addition, we carried out Monte Carlo simulations to model how the exciton diffusion in the semiconductors depends on both the energetic disorder and the concentration of non-radiative quenching-sites. The results are discussed within the context of the diffusion-limited exciton quenching model.

\section{Experimental}

\section{Materials and thin film preparation}

Super Yellow (SY-PPV) was purchased from Merck KGaA $\left(\mathrm{PDY}-132, M_{\mathrm{w}}>1300000 \mathrm{~g} \mathrm{~mol}^{-1}, M_{\mathrm{n}}>400000 \mathrm{~g} \mathrm{~mol}^{-1}\right)$ and phenyl- $\mathrm{C}_{61}$-butyric acid methyl ester (PCBM) was purchased from Solene BV. Both were used without further purification. Poly[2,5-bis(2'-ethylhexyloxy)-1,4-phenylene vinylene] (BEH-PPV) was synthesized in house as follows: $8.76 \mathrm{~g}(16.8 \mathrm{mmol})$ 1,4-bis(bromomethyl)-2,5-bis(2-ethyl-hexyloxy)benzene (premonomer) was dissolved in $500 \mathrm{~mL}$ dry, degassed tetrahydrofuran (THF) in a $1 \mathrm{~L}$ three necked flask and cooled down to $-95{ }^{\circ} \mathrm{C}$ under Ar. In a second flask a base solution of $9.28 \mathrm{~g}$ (82.7 mmol) potassium tert-butoxide was dissolved in $55 \mathrm{~mL}$ dry, degassed THF and also cooled down to $-95{ }^{\circ} \mathrm{C}$ under Ar. Under vigorous stirring, the base solution was added to the 
a)

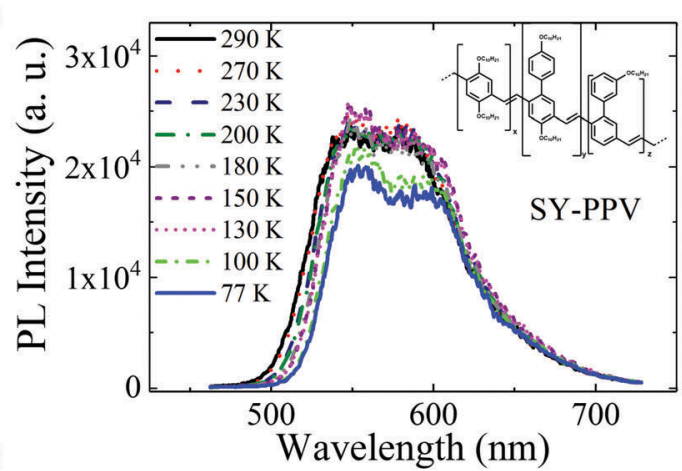

c)

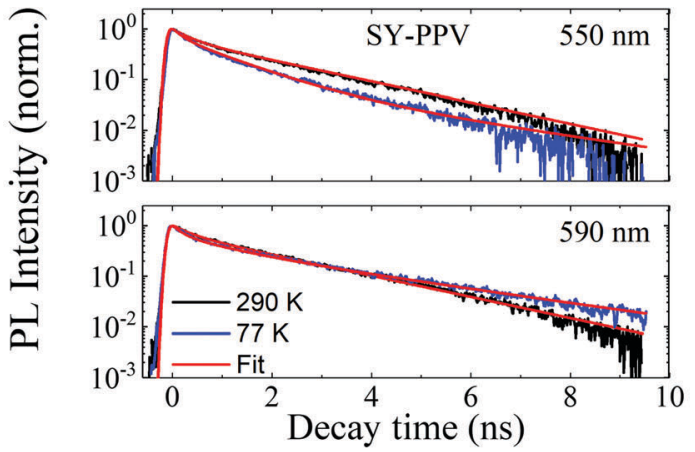

b)

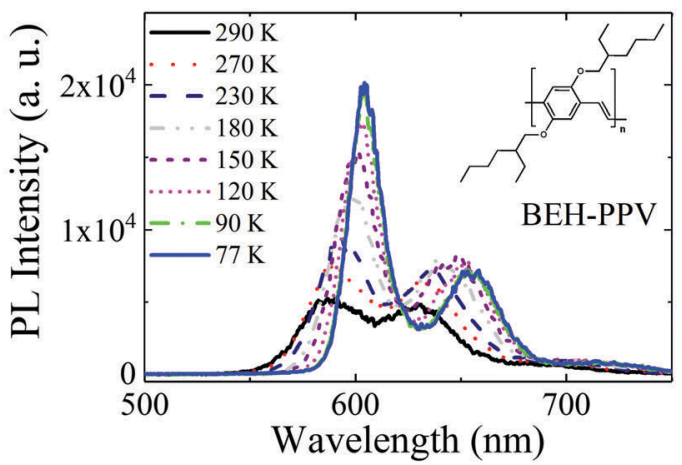

d)

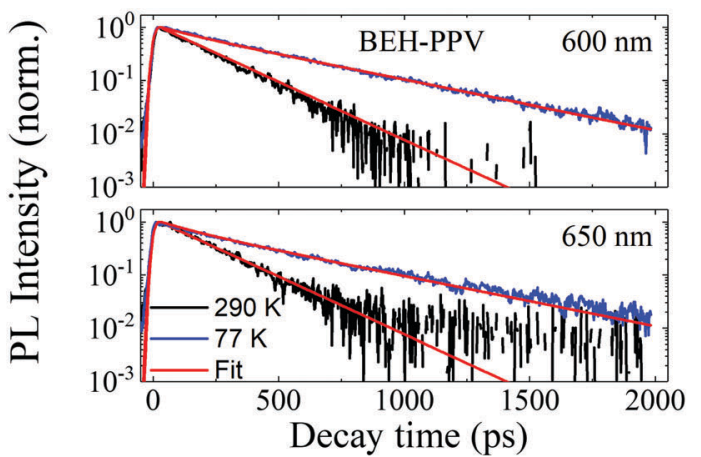

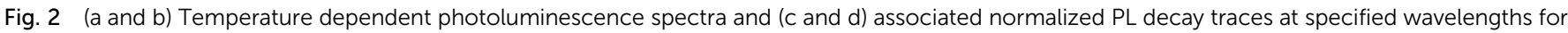

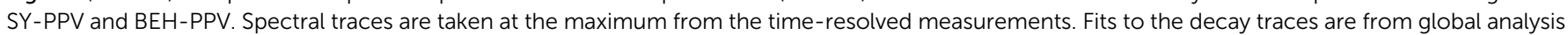
as described in the text, and are further detailed in Tables 1 and 2.

premonomer solution via syringe in order to maintain the $\mathrm{Ar}$ atmosphere. The reaction mixture was left to warm up to room temperature over 5 hours. After 24 hours of total stirring time, the flask was equipped with a reflux condenser and the reaction mixture heated to reflux for 24 hours. Subsequently the polymer was precipitated in $3 \mathrm{~L}$ methanol, filtered off and dried. The resultant solid was dissolved in $700 \mathrm{~mL}$ degassed chloroform to give a concentration of $7 \mathrm{mg} \mathrm{mL}{ }^{-1}$. The polymer solution was again heated to reflux for 48 hours, precipitated in methanol, filtered off, and dried. $4.66 \mathrm{~g}$ of a red compact solid was obtained with $77 \%$ yield. GPC-MALLS (THF, $30{ }^{\circ} \mathrm{C}$ ): $M_{\mathrm{w}}=640370 \mathrm{~g} \mathrm{~mol}^{-1}, M_{\mathrm{n}}=293120 \mathrm{~g} \mathrm{~mol}^{-1}$, PDI $=2.18$.

Thin films for optical characterization were prepared from $6 \mathrm{mg} \mathrm{mL}{ }^{-1}$ (SY-PPV) and $5.2 \mathrm{mg} \mathrm{mL}^{-1}$ (BEH-PPV) polymer or polymer:PCBM blend solution in chlorobenzene (Sigma-Aldrich, anhydrous $99.8 \%$ ) on $1 \times 2 \mathrm{~cm}$ quartz substrates. The substrates were cleaned with acetone and then propanol in an ultrasonic bath and dried for 20 minutes at $140{ }^{\circ} \mathrm{C}$. The films were spin-coated (60 s $1000 \mathrm{rpm} ; 30 \mathrm{~s} 4000 \mathrm{rpm})$ in a nitrogen atmosphere. The polymer layer thicknesses were determined by using a surface profilometer (Bruker, DektakXT), yielding $80 \mathrm{~nm}$ for SY-PPV and $160 \mathrm{~nm}$ for BEH-PPV. The films were measured the same day they were prepared, in order to limit polymer degradation.

\section{Steady-state absorption and photoluminescence}

Steady-state absorption was measured on a Perkin-Elmer Lambda $900 \mathrm{UV} / \mathrm{VIS} / \mathrm{NIR}$ spectrometer. Steady-state photoluminescence was measured on a J\&M Tidas FL3095SL spectrofluorometer.

\section{Time-resolved photoluminescence measurements}

TRPL was measured with a C5680 Hamamatsu streak camera system. Optical samples were photoexcited at $\lambda_{\mathrm{ex}}=400 \mathrm{~nm}$ with the frequency-doubled output from a Ti:sapphire laser (Coherent, Libra HE, $3.5 \mathrm{~mJ}, 100 \mathrm{fs}$ ) with a repetition rate of either $1 \mathrm{kHz}$ (10 ns time window, $0.12 \mathrm{~ns}$ instrument response) or $80 \mathrm{MHz}$ (2 ns time window, $14 \mathrm{ps}$ instrument response). The laser power at the sample was $65 \mu \mathrm{W}$ at $1 \mathrm{kHz}$ for SY-PPV, and $225 \mu \mathrm{W}$ at $80 \mathrm{MHz}$ for BEH-PPV, as these powers avoided annihilation effects (Fig. S1, ESI $\dagger$ ). The laser fluence at the sample was $<1 \mu \mathrm{J} \mathrm{cm}^{-2}$. In order to limit polymer degradation due to oxygen exposure, the samples were loaded into a continuous flow nitrogen cooled cryostat (Optistat CF-V2, Oxford) equipped with temperature controller (Mercury iTC, Oxford) and measured under a dynamic vacuum of $10^{-4}-10^{-5} \mathrm{mbar}$ for the entire temperature range. Each sample was first measured at $298 \mathrm{~K}$ and then cooled to $77 \mathrm{~K}$. The sample was then heated to each measurement temperature at a rate of $\sim 1 \mathrm{~K} \mathrm{~min}^{-1}$, and the temperature stabilized for 5 minutes before running the experiment. At the end of the heating procedure, the measurement at $298 \mathrm{~K}$ was repeated. The consistency of the $298 \mathrm{~K}$ measurements provided good evidence that the sample did not intrinsically change over the course of the experiments.

\section{Data analysis and simulations}

The photoluminescence decay dynamics are derived from global analysis $^{19}$ of the TRPL data was done using the R-package 
TIMP software ${ }^{20}$ with the graphical interface Glotaran 1.5.1. ${ }^{21}$ Regarding the Monte Carlo simulation, the utilized scheme models the PL decay in semiconductor-quencher mixtures in order to extract the exciton diffusion parameters, where the excitons and the quencher molecules, depending on their concentration, are randomly placed in a cubic simulation box of $50 \times 50 \times 50 \mathrm{~nm}$ length as balls of $1 \mathrm{~nm}$ diameter. The simulation supposes normal diffusion, which is modeled as a random walk with a constant exciton hopping distance ("hop size"). For each time iteration $\delta t$ every exciton is moved in a random 3D direction for a fixed distance $\delta s$, which correlates with the exciton diffusion coefficient as follows: $D=\delta s^{2} / 6 \delta t$. Radiative recombination is assumed if an exciton has not been quenched after time $\tau_{i}$, which is fixed at the beginning of the simulation. Thus, the simulation is based on the exciton-diffusion limited quenching model at non-radiative quenchers. A comprehensive description of the Monte Carlo simulation is described elsewhere, ${ }^{22,23}$ and the software package can be downloaded from the internet. ${ }^{24}$ In the case of a monoexponential PL decay, the Stern-Volmer analysis is used to extract the exciton diffusion parameters, as described previously in literature. ${ }^{12,14,15}$

\section{PLED device fabrication and characterization}

The PLED devices were fabricated on glass-ITO (tin-doped indium oxide) substrates according to the device structure ITO/PEDOT:PSS (55 nm)/PPV/Ba (5 nm)/Al (100 nm). The substrates were cleaned with acetone and propanol in an ultrasonic bath, dried and treated with ultraviolet (UV)-ozone. PEDOT:PSS (Heraeus Clevios $^{\mathrm{TM}}$ Al 4083) was spin-coated (60 s 1200 rpm; $20 \mathrm{~s} 4000 \mathrm{rpm}$ ) after UV-ozone treatment. The PPV layers were spin-coated in a nitrogen atmosphere. The layer thickness of BEH-PPV was $165 \mathrm{~nm}$, obtained from $5.2 \mathrm{mg} \mathrm{mL}^{-1}$ chlorobenzene solution and spin-speeds of $1000 \mathrm{rpm}$ for $60 \mathrm{~s}$ followed by $4000 \mathrm{rpm}$ for $30 \mathrm{~s}$. The layer thickness of SY-PPV was $80 \mathrm{~nm}$, obtained from a $6 \mathrm{mg} \mathrm{mL} \mathrm{mL}^{-1}$ chlorobenzene solution $(60 \mathrm{~s}$ $1000 \mathrm{rpm} ; 30 \mathrm{~s} 4000 \mathrm{rpm})$. The SY-PPV device fabricated from a $5.5 \mathrm{mg} \mathrm{mL}{ }^{-1}$ toluene solution (20 s $1000 \mathrm{rpm}$; $60 \mathrm{~s} 250 \mathrm{rpm}$ ), gave a film thickness of $100 \mathrm{~nm}$. The $\mathrm{Ba} / \mathrm{Al}$ cathode was thermally evaporated onto the PPV layer (chamber pressure $10^{-7} \mathrm{mbar}$ ). The current efficiencies were measured with a Keithley 2400 source meter in nitrogen atmosphere. The electroluminescence spectra were measured at $7 \mathrm{~V}$ with an Ocean optics (USB4000-UV-Vis-ES) spectrometer.

\section{Results}

\section{Temperature-dependent time-resolved photoluminescence}

The PL spectrum of SY-PPV collected at the maximum of the TRPL intensity (Fig. 2a) exhibits a broad line shape from 500-700 nm, which fits to a sum of three Gaussian peaks (Table S1, ESI $\dagger$ ), ascribed to the electronic $\mathrm{S}_{0,0}$ and vibronic $S_{0,1}$ and $S_{0,2}$ transitions. The peak positions are in good agreement with previous reports, ${ }^{25}$ as well as the steady-state PL measurements (Fig. S2, ESI $\dagger$ ). The PL spectrum red-shifts with lowering temperature. The peak intensity stays relatively constant from 290-130 K, and then slightly decreases at 100 and $77 \mathrm{~K}$. The PL spectrum of BEH-PPV spans 550-750 nm (Fig. 2b) and is similarly characterized by one primarily electronic transition $\left(\mathrm{S}_{0,0}\right)$ and two vibronic peaks $\left(\mathrm{S}_{0,1}, \mathrm{~S}_{0,2}\right)$. The peak positions from a fit to a sum of three Gaussians are shown in Table S2 (ESI $\dagger$ ). With lowering temperature, the PL spectrum of BEH-PPV red-shifts and the peak intensity increases. The relative intensity between the electronic $\left(\mathrm{S}_{0,0}\right)$ and vibronic peaks $\left(\mathrm{S}_{0, x}\right)$ also changes with decreasing temperature. At $298 \mathrm{~K}$, the $S_{0,0}$ and $S_{0,1}$ transitions are of similar intensity, while at $77 \mathrm{~K}$, the $S_{0,0}$ transition is more than twice as intense as the $S_{0,1}$ transition, in agreement with previous work. This indicates a decrease in electron-vibrational coupling with decreasing temperature. ${ }^{26}$ The narrowing of the fluorescence peaks with lowering temperature is more evident in BEH-PPV as opposed to SY-PPV.

The comparison of the normalized PL decay kinetics measured at the $S_{0,0}(550 \mathrm{~nm})$ and $S_{0,1}(590 \mathrm{~nm})$ transitions (Fig. 2c, black and blue traces) demonstrate that the SY-PPV temperaturedependence depends on the emission wavelength. At $550 \mathrm{~nm}$, the $77 \mathrm{~K}$ trace decays faster than the $290 \mathrm{~K}$ trace, while at $590 \mathrm{~nm}$, the traces are mostly similar, with the $290 \mathrm{~K}$ trace decaying faster by a small amount. Fig. 2c shows only the lowest and highest measured temperatures for the $S_{0,0}$ and $S_{0,1}$ transition, for clarity, but the observed trend is consistent throughout the entire measured temperature range (Fig. S3, ESI $\dagger$ ). The normalized BEH-PPV PL decay traces (Fig. 2d), monitored at 600 and $650 \mathrm{~nm}$, demonstrate that the PL lifetime at $77 \mathrm{~K}$ is longer than at $290 \mathrm{~K}$ at all wavelengths. This trend is consistent through the measured temperature range (Fig. S4, ESI $\dagger$ ).

The TRPL decay dynamics were modeled with a global analysis scheme, which takes into account the decay dynamics across the entire spectral range. ${ }^{19,21}$ The fits are represented as red lines in Fig. $2 \mathrm{c}$ and $\mathrm{d}$ and the lifetimes and relative amplitudes are summarized in Tables 1 and 2. For both materials, the data were fit to a sum of a fast and slow exponential contribution. The measured exciton lifetime $\left(\tau_{\mathrm{f}}\right)$ is then determined from a weighted average of the two kinetic components, (eqn (3))

$$
\tau_{\mathrm{av}}=\langle t\rangle=\frac{\int_{0}^{\infty} t I(t) \mathrm{d} t}{\int_{0}^{\infty} I(t) \mathrm{d} t}=\frac{\sum_{i} a_{i} \tau_{i}^{2}}{\sum_{i} a_{i} \tau_{i}}
$$

and the relative PL quantum yields $\left(\phi_{\mathrm{T}} / \phi_{290 \mathrm{~K}}\right)$ are determined from the integral under the spectrum. In SY-PPV (Table 1), both the fast and slow lifetimes become longer with decreasing temperature, which is attributed to a decrease in non-radiative contributions. Simultaneously, the relative amplitude of the fast component increases with decreasing temperature. This is consistent with an increase in non-radiative processes due to energy transfer/hopping to conformational subunits exhibiting longer decay rates. ${ }^{25}$ These varying contributions to the non-radiative PL decay result in an overall slight increase in the average PL lifetime, $\tau_{\mathrm{f}}$, from $1.9 \mathrm{~ns}$ at $290 \mathrm{~K}$ to $2.4 \mathrm{~ns}$ at $77 \mathrm{~K}$. The relative quantum yield does not vary significantly with temperature 
Table 1 Temperature dependence of the average PL lifetime $\tau_{f}$ and calculated relative quantum yield $\phi_{T} / \phi_{290 K}$ in SY-PPV

\begin{tabular}{lllllll}
\hline$T(\mathrm{~K})$ & $A_{1}$ & $\tau_{1}(\mathrm{ps})$ & $A_{2}$ & $\tau_{2}(\mathrm{ps})$ & $\tau_{\mathrm{f}}(\mathrm{ps})$ & $\phi_{\mathrm{T}} / \phi_{290 \mathrm{~K}}$ \\
\hline 290 & 0.39 & 360 & 0.61 & 2080 & 1900 & 1.00 \\
270 & 0.42 & 370 & 0.58 & 2190 & 1990 & 1.02 \\
230 & 0.46 & 410 & 0.54 & 2390 & 2140 & 0.99 \\
200 & 0.47 & 420 & 0.53 & 2480 & 2200 & 0.98 \\
180 & 0.49 & 440 & 0.51 & 2540 & 2240 & 0.96 \\
150 & 0.51 & 450 & 0.49 & 2570 & 2250 & 1.01 \\
130 & 0.51 & 460 & 0.49 & 2600 & 2260 & 0.99 \\
100 & 0.53 & 480 & 0.47 & 2680 & 2320 & 0.84 \\
77 & 0.51 & 490 & 0.49 & 2710 & 2360 & 0.78
\end{tabular}

Table 2 Temperature dependence of the average PL lifetime $\tau_{\mathrm{f}}$ and calculated relative quantum yield $\phi_{\mathrm{T}} / \phi_{290 \mathrm{~K}}$ in $\mathrm{BEH}-\mathrm{PPV}$

\begin{tabular}{lllllll}
\hline$T(\mathrm{~K})$ & $A_{1}$ & $\tau_{1}(\mathrm{ps})$ & $A_{2}$ & $\tau_{2}(\mathrm{ps})$ & $\tau_{\mathrm{f}}(\mathrm{ps})$ & $\phi_{\mathrm{T}} / \phi_{290 \mathrm{~K}}$ \\
\hline 290 & 0.89 & 160 & 0.11 & 370 & 210 & 1.00 \\
270 & 0.91 & 180 & 0.09 & 450 & 240 & 1.32 \\
230 & 0.90 & 220 & 0.10 & 550 & 290 & 1.49 \\
180 & 0.87 & 270 & 0.13 & 670 & 370 & 1.70 \\
150 & 0.83 & 290 & 0.17 & 630 & 390 & 1.84 \\
120 & 0.79 & 300 & 0.21 & 600 & 410 & 1.76 \\
90 & 0.62 & 190 & 0.38 & 480 & 370 & 1.71 \\
77 & 0.56 & 130 & 0.44 & 480 & 390 & 1.76
\end{tabular}

until $130 \mathrm{~K}$. The reduction of $\phi_{\mathrm{f}}$ at temperatures $<130 \mathrm{~K}$ is attributed to an increased energy transfer to non-luminescent sub-species due to increased interchain interactions at low temperatures. ${ }^{10,11,27}$ In BEH-PPV (Table 2), the two-component model was required to account for energy transfer from shorter to longer conformational subunits, which contributes more with decreasing temperature. Both the fast and slow components get longer with decreasing temperature until $150 \mathrm{~K}$. In this case, the relative amplitude of the slow component increases with decreasing temperature. As a result, the average lifetime $\tau_{\mathrm{f}}$ increases from $207 \mathrm{ps}$ at $290 \mathrm{~K}$ to $405 \mathrm{ps}$ at $150 \mathrm{~K}$. At temperatures $<150 \mathrm{~K}$, the decay constant $\tau_{1}$ becomes faster, probably due to more contribution from energy transfer and interchain quenching interactions. ${ }^{10,11}$ The relative quantum yield $\phi_{\mathrm{T}} / \phi_{290 \mathrm{~K}}$ of BEH-PPV also increases with decreasing temperature. The concomitant increase in lifetime and quantum yield with decreasing temperature indicates that in BEH-PPV the PL is predominantly radiative, where lower temperatures result in a decrease in competitive non-radiative processes, consistent with the observed decrease in electron-vibrational coupling.

\section{Exciton diffusion coefficient and diffusion length}

As a next step, we investigated the spatial and temporal evolution of the singlet exciton population, characterized by the exciton diffusion coefficient $D$, as a function of temperature. We measured fluorescence quenching dynamics of thin polymer films with randomly distributed PCBM molecules, known to be efficient exciton quenchers. ${ }^{9,14,28-30}$ Thin films of SY-PPV and BEH-PPV, each blended with known concentrations of PCBM, were prepared from chlorobenzene solution. The average fluorescence lifetime was determined from TRPL experiments, using the same procedure described in the previous section.
The results were analyzed by Monte Carlo simulation, which is appropriate for multi-exponential decays, to return the exciton diffusion coefficient $D .^{22,23}$ The exciton diffusion length $L_{\mathrm{D}}$, which corresponds to the average distance an exciton can diffuse during its lifetime $\tau_{\mathrm{f}}$, is calculated as follows,

$$
L_{\mathrm{D}}=\sqrt{\tau_{\mathrm{f}} D}
$$

where $\tau_{\mathrm{f}}$ is the average lifetime calculated from the TRPL measurements (Tables 1 and 2). The results are shown in Fig. 3 and further detailed in Table S3 (ESI $\dagger$ ).

The better ordered BEH-PPV exhibits overall higher exciton diffusion coefficients and exciton diffusion lengths than the less well-ordered SY-PPV. With lowering temperature, SY-PPV shows a significant reduction of the exciton diffusion parameters between 298-180 K, with $D$ decreasing from $3.1 \times 10^{-4} \mathrm{~cm}^{2} \mathrm{~s}^{-1}$ to $8.0 \times 10^{-5} \mathrm{~cm}^{2} \mathrm{~s}^{-1}$ and $L_{\mathrm{D}}$ decreasing from $7.7 \mathrm{~nm}$ to $4.2 \mathrm{~nm}$. Further cooling from $180 \mathrm{~K}$ to $77 \mathrm{~K}$ reveals nearly constant exciton diffusion parameters for SY-PPV. In contrast, the exciton diffusion parameters for the better ordered BEH-PPV display less temperature dependence in the higher temperature range (298-120 K), while in the lower temperature range 100-77 K, there is greater dependence on temperature, with $D$ decreasing from $2.5 \times 10^{-3} \mathrm{~cm}^{2} \mathrm{~s}^{-1}$ to $6.8 \times 10^{-4} \mathrm{~cm}^{2} \mathrm{~s}^{-1}$ and $L_{\mathrm{D}}$ decreasing from $9.6 \mathrm{~nm}$ to $5.2 \mathrm{~nm}$. Both polymers exhibit an approximately $75 \%$ reduction in $D$ at $77 \mathrm{~K} v s .290 \mathrm{~K}$.

For BEH-PPV, we also compared these results with values derived from a monoexponential fit to the fluorescence decay, which we have reported before for this polymer at room temperature. ${ }^{14}$ The temperature-dependent exciton diffusion parameters, in this case determined by the Stern-Volmer equation, and exciton lifetimes determined from monoexponential fits of the PL decays are shown in Fig. S5 (ESI $\dagger$ ). The results show a similar trend of $D$ and $L_{\mathrm{D}}$ in BEH-PPV compared to the results determined from global analysis and Monte Carlo simulations (Fig. 3), with slightly higher values for $L_{\mathrm{D}}$. As $D$ is quite similar for the two fits, the difference in $L_{\mathrm{D}}$ is most likely due to the longer fluorescence lifetime from the monoexponential fits, which do not account for the increased energy transfer between polymer chains at lower temperatures.

\section{Temperature-dependent polymer light-emitting diode efficiencies}

PLEDs of each SY-PPV and BEH-PPV were fabricated in order to test the temperature dependence of the device efficiencies. The device structure ITO/PEDOT:PSS/PPV/Ba/Al was used for both active layer materials. The SY-PPV active layer is $80 \mathrm{~nm}$ thick and the BEH-PPV layer is $165 \mathrm{~nm}$ thick. Fig. 4 shows the temperature dependent device current efficiencies $v s$. voltage. The current efficiency is defined as photon/charge carrier. It is calculated by dividing the photocurrent density by the electrical current density (Fig. S6, ESI $\dagger$ ). This unitless value is a measure for the relation between the number of emitted photons and the number of charges that pass through the device for a given time interval. The SY-PPV PLED exhibits no significant change in current efficiency with decreasing temperature in the range 
a)
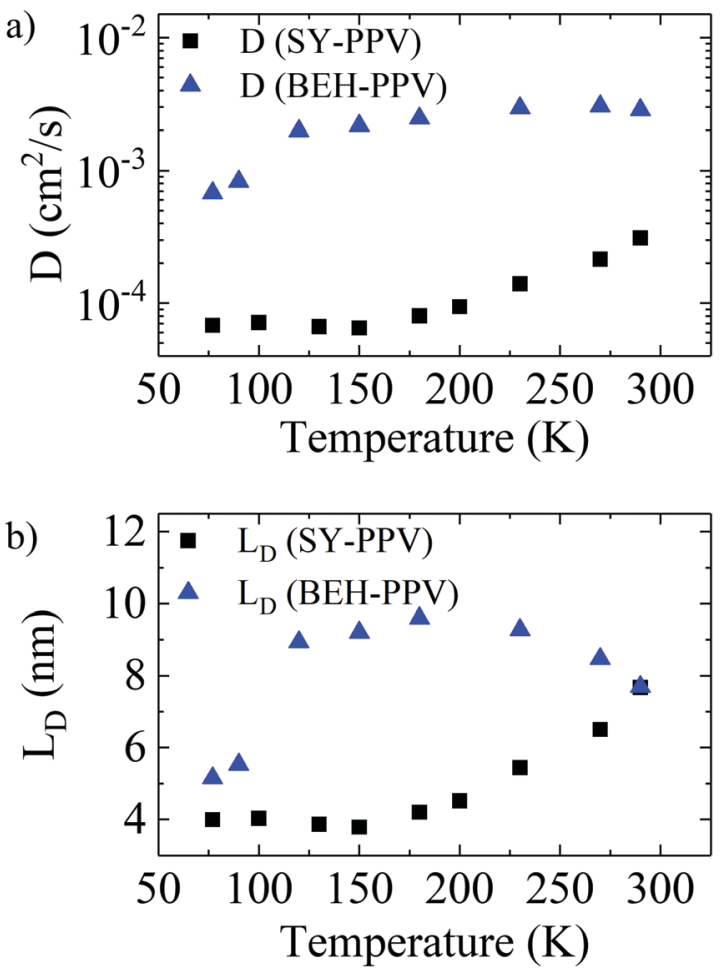

Fig. 3 Temperature-dependence of (a) exciton diffusion coefficient $D$ and (b) exciton diffusion length $L_{D}$ for SY-PPV and BEH-PPV, determined from time-resolved fluorescence quenching experiments of thin films. of $295-233 \mathrm{~K} .{ }^{17}$ We also confirmed this in a device made from toluene, which is the more commonly used solvent for processing SY-PPV ${ }^{31}$ (Fig. S7, ESI $\dagger$ ). The current efficiency of the BEH-PPV PLED, however, increases by more than a factor of two when the device temperature is decreased between 295-215 K. Additionally, the rise of current efficiency with increasing voltage becomes steeper as the BEH-PPV device temperature is lowered.

Temperature dependent EL spectra of SY-PPV and BEH-PPV are shown in Fig. $4 \mathrm{c}$ and d. The SY-PPV EL appears qualitatively similar to the PL, while the BEH-PPV EL demonstrates a different spectral line shape than PL spectra in the same temperature range, with the $\mathrm{S}_{0,0}$ peak exhibiting always higher intensity than $\mathrm{S}_{0,1}$. To understand this further, we compared the steady-state PL of the pristine polymer films to that within the PLED stack (Fig. S2, ESI $\dagger$ ). Under the same measurement conditions, the BEH-PPV PL spectrum within the PLED stack shows a change in peak ratio relative to the pristine BEH-PPV film. In SY-PPV, there is also a small shift and narrowing of the PL spectrum within the PLED stack. This EL spectral line shape is consistent with an optical microcavity effect in the PLED, wherein the near-field coupling results in a narrowing of the spectral line shape and enhances the spontaneous emission rate. ${ }^{2,32}$ Our results in Fig. 4 and Fig. S2 (ESI $\dagger$ ) show that this is a much stronger effect in the $165 \mathrm{~nm}$ BEH-PPV device than in the $80 \mathrm{~nm}$ SY-PPV device. a)

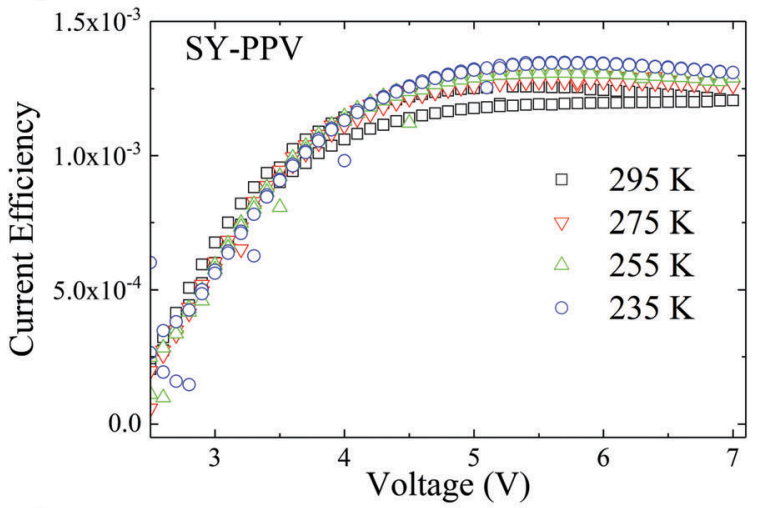

c)

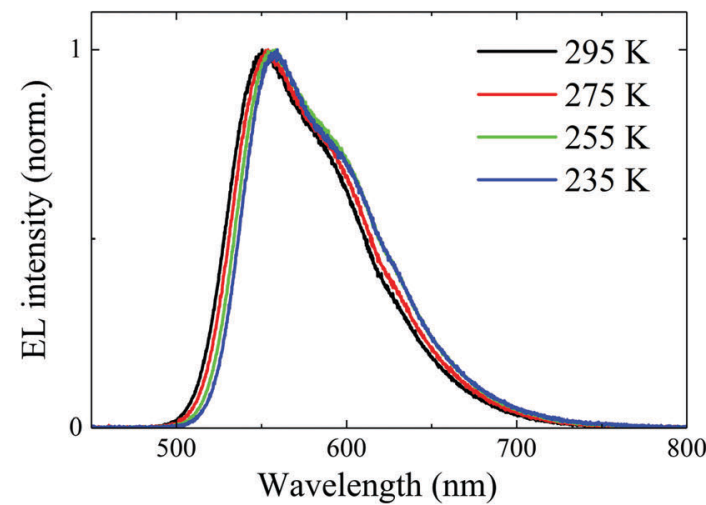

b)

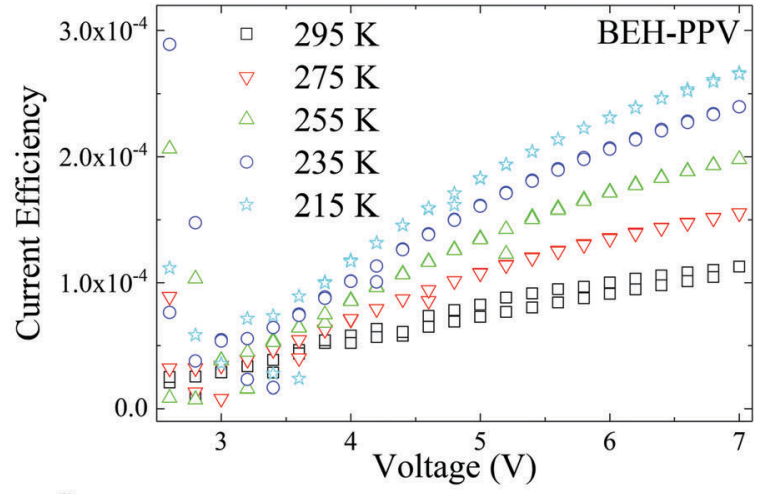

d)

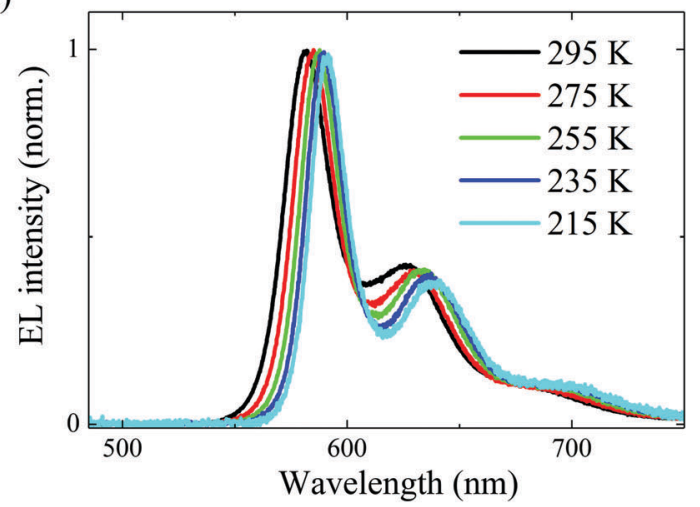

Fig. 4 Temperature dependent current efficiencies vs. voltage of PLEDs comprised of (a) SY-PPV and (b) BEH-PPV along with electroluminescence spectra of the (c) SY-PPV and (d) BEH-PPV PLED measured at $7 \mathrm{~V}$, normalized to the spectral maximum. 


\section{Discussion}

The BEH-PPV and SY-PPV polymers studied here have different DOS characteristics due in part to their molecular conformations. The chemical structures in Fig. 2 show that BEH-PPV has two symmetrical alkoxy side chains and the configurational freedom of the individual chains is limited, leading to less energy spread of the Gaussian density of states, ${ }^{33}$ and thus a narrow width of the energy distribution. In contrast, the random copolymer SY-PPV exhibits high configurational freedom of individual conformational subunits, resulting in more structural disorder and thus a broader width of the Gaussian DOS. The width of the excitonic DOS $\left(\sigma_{\text {optical }}\right)$ is calculated by assuming that the energy position of the $S_{0,0}$ transition from our PL measurements corresponds to the level of the most populated states, which lies at the equilibrium level $-\sigma^{2} / k T$ below the center of the DOS. ${ }^{1,9}$ The slope of the relationship between the $\mathrm{S}_{0,0}$ peak position vs. $1 / T$ allows us to extract $\sigma_{\text {optical }}$. From this we find that energetic disorder of BEH-PPV $\left(\sigma_{\text {optical }}=0.033 \mathrm{eV}\right)$ is indeed less than that of SY-PPV $\left(\sigma_{\text {optical }}=\right.$ $0.048 \mathrm{eV}$ ). The half-width of the excitonic (optical) Gaussian DOS is about 3 times smaller than the half-width of the charge carrier (electrical) DOS extracted from charge transport measurements, for BEH-PPV $\sigma_{\text {electrical }}=0.092 \mathrm{eV}$ and SY-PPV $\sigma_{\text {electrical }}=0.140 \mathrm{eV}$, respectively. ${ }^{16}$ Previous work showed that $\sigma_{\text {electrical }}$ is larger than $\sigma_{\text {optical }}$ due to the different nature of interactions between charge carriers and excitons within the DOS. ${ }^{1}$ Nonetheless, BEH-PPV reproducibly exhibits a narrower DOS than SY-PPV.

Singlet excited states in disordered polymers are generated at an arbitrary energy-site within the high-energy tail of the Gaussian DOS and simultaneously undergo downhill migration toward lower energy-sites. ${ }^{7,34-36}$ The down-hill migration, also known as spectral diffusion, ends when the excitons approach the energy level of the most populated excited states, provided that the time needed to approach quasi-equilibrium is shorter than the intrinsic exciton lifetime. At room temperature, spectral diffusion is followed by temperature activated hopping, where balanced downward and thermally activated upward hopping occurs. With decreasing temperature, the equilibrium level shifts deeper into the tail of the Gaussian DOS, causing a redshift of the PL spectrum. ${ }^{9,25,36}$ There, the probability to transfer to nearby energy-sites decreases, resulting in a slower exciton transfer. Additionally, thermally activated hopping becomes less pronounced. Thus, the exciton diffusion characteristics (and quantum yield) of a conjugated polymer thin film depend strongly on the disorder parameter $\sigma$ and the temperature $T,{ }^{37}$ as well as on the starting singlet excitation energy within the DOS and the coupling strength between the energy sites. ${ }^{38}$

\section{Temperature-dependent exciton decay characteristics}

The differences in energetic disorder between BEH-PPV and SY-PPV manifest in the temperature dependent PL and exciton diffusion characteristics as measured in thin films. The exciton decay processes in these systems are governed by radiative decay, non-radiative decay, and exciton diffusion to non-radiative
Table 3 Exciton decay and transfer rates in SY-PPV, using an absolute PLQY of $60 \%$ for the pristine film at $290 \mathrm{~K}^{27}$

\begin{tabular}{llllll}
\hline$T(\mathrm{~K})$ & $\phi_{\mathrm{f}}$ & $k_{\mathrm{f}}\left(\mathrm{s}^{-1}\right)$ & $k_{\mathrm{r}}\left(\mathrm{s}^{-1}\right)$ & $k_{\text {diff }}\left(\mathrm{s}^{-1}\right)$ & $k_{\mathrm{nr}}\left(\mathrm{s}^{-1}\right)$ \\
\hline 290 & 0.60 & $5.3 \times 10^{8}$ & $3.2 \times 10^{8}$ & $5.2 \times 10^{7}$ & $1.6 \times 10^{8}$ \\
270 & 0.61 & $5.0 \times 10^{8}$ & $3.1 \times 10^{8}$ & $3.6 \times 10^{7}$ & $1.6 \times 10^{8}$ \\
230 & 0.59 & $4.7 \times 10^{8}$ & $2.8 \times 10^{8}$ & $2.3 \times 10^{7}$ & $1.7 \times 10^{8}$ \\
200 & 0.59 & $4.5 \times 10^{8}$ & $2.7 \times 10^{8}$ & $1.5 \times 10^{7}$ & $1.7 \times 10^{8}$ \\
180 & 0.57 & $4.5 \times 10^{8}$ & $2.6 \times 10^{8}$ & $1.3 \times 10^{7}$ & $1.8 \times 10^{8}$ \\
150 & 0.60 & $4.5 \times 10^{8}$ & $2.7 \times 10^{8}$ & $1.1 \times 10^{7}$ & $1.7 \times 10^{8}$ \\
130 & 0.60 & $4.4 \times 10^{8}$ & $2.6 \times 10^{8}$ & $1.1 \times 10^{7}$ & $1.7 \times 10^{8}$ \\
100 & 0.51 & $4.3 \times 10^{8}$ & $2.2 \times 10^{8}$ & $1.1 \times 10^{7}$ & $2.0 \times 10^{8}$ \\
77 & 0.47 & $4.2 \times 10^{8}$ & $2.0 \times 10^{8}$ & $1.1 \times 10^{7}$ & $2.1 \times 10^{8}$ \\
& & & & &
\end{tabular}

Table 4 Exciton decay and transfer rates in BEH-PPV, using an absolute PLQY of $6 \%$ for the pristine film at $290 \mathrm{~K}$

\begin{tabular}{llllll}
\hline$T(\mathrm{~K})$ & $\phi_{\mathrm{f}}$ & $k_{\mathrm{f}}\left(\mathrm{s}^{-1}\right)$ & $k_{\mathrm{r}}\left(\mathrm{s}^{-1}\right)$ & $k_{\text {diff }}\left(\mathrm{s}^{-1}\right)$ & $k_{\mathrm{nr}}\left(\mathrm{s}^{-1}\right)$ \\
\hline 290 & 0.06 & $5.0 \times 10^{9}$ & $3.0 \times 10^{8}$ & $4.3 \times 10^{9}$ & $3.6 \times 10^{8}$ \\
270 & 0.08 & $4.7 \times 10^{9}$ & $3.7 \times 10^{8}$ & $4.1 \times 10^{9}$ & $2.1 \times 10^{8}$ \\
230 & 0.09 & $3.9 \times 10^{9}$ & $3.5 \times 10^{8}$ & $3.4 \times 10^{9}$ & $1.4 \times 10^{8}$ \\
180 & 0.10 & $2.9 \times 10^{9}$ & $2.9 \times 10^{8}$ & $2.5 \times 10^{9}$ & $1.3 \times 10^{8}$ \\
150 & 0.11 & $2.7 \times 10^{9}$ & $2.9 \times 10^{8}$ & $2.2 \times 10^{9}$ & $1.4 \times 10^{8}$ \\
120 & 0.11 & $2.9 \times 10^{9}$ & $3.0 \times 10^{8}$ & $2.4 \times 10^{9}$ & $2.0 \times 10^{8}$ \\
90 & 0.10 & $2.7 \times 10^{9}$ & $2.8 \times 10^{8}$ & $1.8 \times 10^{9}$ & $6.5 \times 10^{8}$ \\
77 & 0.11 & $2.7 \times 10^{9}$ & $2.9 \times 10^{8}$ & $1.7 \times 10^{9}$ & $7.5 \times 10^{8}$ \\
\hline
\end{tabular}

traps (Fig. 1). The TRPL experiments show that, with decreasing temperature, both the SY-PPV and BEH-PPV films demonstrate longer fluorescence lifetimes. However, while the relative quantum yield for SY-PPV is fairly constant until very low temperatures, the one of BEH-PPV increases along with the lifetime.

The excited state transfer rates for the two polymers were calculated in order to quantitatively describe the exciton decay processes (Tables 3, 4 and Fig. S8, ESI $\dagger$ ). The lifetime $\tau_{i}$ correlates with the transfer rate $k_{i}$ with the following relation: $\tau_{i}=1 / k_{i}$. The values were determined as follows: the radiative decay rate $k_{\mathrm{r}}$ is first calculated by eqn (5),

$$
k_{\mathrm{r}}=\phi_{\mathrm{f}} / \tau_{\mathrm{f}}=\left(\phi_{\mathrm{T}} / \phi_{290 \mathrm{~K}} \cdot \phi_{\mathrm{abs}}\right) / \tau_{\mathrm{f}}
$$

where $\phi_{\mathrm{f}}$ corresponds to the product of the relative PLQY $\left(\phi_{\mathrm{T}} / \phi_{290 \mathrm{~K}}\right)$ and the absolute PLQY $\left(\phi_{\mathrm{abs}}\right)$ of a pristine film at $290 \mathrm{~K}$. We used the literature reported $\phi_{\mathrm{abs}}=60 \%$ for an SY-PPV film, ${ }^{27}$ which returns a natural exciton lifetime $\tau_{\mathrm{r}}=1 / k_{\mathrm{r}} \sim 3 \mathrm{~ns}$. From the measured fluorescence lifetimes at $290 \mathrm{~K}$, the absolute PLQY of our BEH-PPV thin film is approximately ten times smaller $\left(\phi_{\mathrm{abs}} \sim 6 \%\right)$ than the one of SY-PPV, also leading to a natural exciton lifetime $\tau_{\mathrm{r}}$ of $\sim 3$ ns. Considering the two materials have the same polymer backbone, a similar natural exciton lifetime for both PPV derivatives is expected. We note that in literature $\phi_{\mathrm{abs}}$ has been reported as high as $12 \%$ for a BEH-PPV film, ${ }^{39}$ which would give rise to a natural exciton lifetime of $\tau_{\mathrm{r}} \sim 1.7 \mathrm{~ns}$. However, a value of $\phi_{\mathrm{abs}}=12 \%$ does not change the trend of the transfer rates in BEH-PPV.

In order to disentangle $k_{\mathrm{nr}}$ from $k_{\text {diff, }}$, we used the exciton diffusion model to simulate quenching efficiencies $Q\left(Q=1-N_{\mathrm{R}} / N_{0}\right)$ for a range of diffusion coefficients $D$. The solid lines in Fig. 5 show the dependence of $Q$ as determined by the ratio of the number of radiatively decaying excitons $\left(N_{\mathrm{R}}\right)$ 
and the number of excitons that would decay radiatively in the absence of a quencher $\left(N_{0}\right)$. The curves represent $Q$ for a constant intrinsic exciton lifetime $\tau_{\mathrm{r}}=3 \mathrm{~ns}$. The model also assumes a constant number of exciton defects (background quenchers). In SY-PPV an electron trap density of $1 \times 10^{17} \mathrm{~cm}^{-3}$ was determined by charge transport measurements, ${ }^{40}$ and in BEH-PPV a trap concentration of $6.5 \times 10^{17} \mathrm{~cm}^{-3}$ was determined by using Stern-Volmer analysis, as described previously. ${ }^{14}$ Fig. 5 shows that $Q$ increases with enhanced $D$, since a high exciton diffusion coefficient represents a fast exciton diffusion toward traps, leading to more efficient exciton quenching, and vice versa. The quenching efficiency is then retrieved from the calculated value of $D$ from TRPL, and $k_{\text {diff }}$ is determined by $k_{\mathrm{f}} \times Q$.

Fig. 5 shows that, at room temperature, almost $90 \%$ of the excitons in BEH-PPV are quenched at a defect, whereas for SY-PPV, this value is less than $10 \%$. As seen in Tables 3 and 4 , $k_{\text {diff }}$ of BEH-PPV is two orders of magnitude higher than that of SY-PPV. As a result, the non-radiative losses in BEH-PPV are almost fully dominated by diffusion-limited quenching at the defects present in the polymer. Furthermore, both PPV derivatives demonstrate a decrease in $k_{\text {diff }}$ with lowering temperature, in agreement with the diffusion limited exciton quenching model. With the values of $k_{\mathrm{r}}$ and $k_{\text {diff }}$ known, the non-radiative transfer rates $k_{\mathrm{nr}}$ were determined using eqn (1) and (2).

The calculated values show that $k_{\mathrm{r}}$ in SY-PPV slowly decreases with decreasing temperature, while in BEH-PPV it barely changes. This is consistent with the predominantly radiative PL observed in BEH-PPV. Considering the non-radiative processes, exciton diffusion towards defects $\left(k_{\text {diff }}\right)$ is largely determining the PL quenching in BEH-PPV (Fig. 5). In contrast, in SY-PPV $k_{\mathrm{nr}}$ is the dominant non-radiative loss process.

The increase of $k_{\mathrm{nr}}$ with a concomitant slight decrease of $k_{\mathrm{r}}$ in SY-PPV is ascribed to increased population of conformational subunits that have higher contribution from non-radiative decay

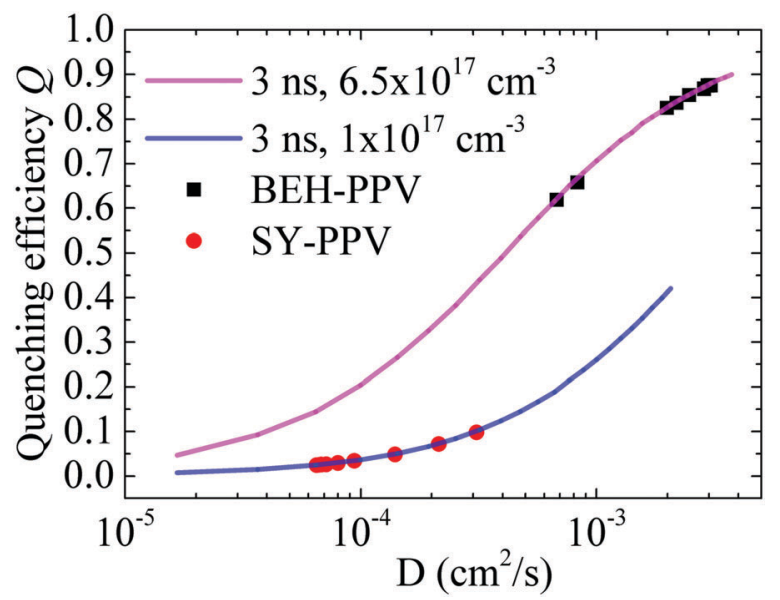

Fig. 5 Exciton diffusion coefficient $D$ dependent quenching efficiency $Q$ for SY-PPV and BEH-PPV. The amount of background quenchers equals $1 \times 10^{17} \mathrm{~cm}^{-3}$ and $6.5 \times 10^{17} \mathrm{~cm}^{-3}$ for SY-PPV and BEH-PPV, respectively. The black and red points correspond to the values of $D$ determined from the experimental results (Fig. 3). processes, such as may be expected with increased interchain interactions. Also, $k_{\text {diff }}$ remains temperature-independent below $180 \mathrm{~K}$. The decrease in the exciton diffusion rate $k_{\text {diff }}$ with lower temperature leads to a slight increase in the overall (average) exciton lifetime from $1.9 \mathrm{~ns}$ to $2.4 \mathrm{~ns}$ going from $290 \mathrm{~K}$ to $77 \mathrm{~K}$. The wavelength dependent exciton lifetimes are attributed to different sub-populations of emitting species with varying degrees of radiative and non-radiative emission, where at higher energies the radiative species dominates the fluorescence. At lower energies, there are greater contributions due to spectral diffusion caused by energy transfer from shorter to longer conformational subunits as well as from energy transfer to interchain excimer like species, which will have a lower oscillator strength and subsequently longer PL lifetime. ${ }^{25,27}$ The exciton decay processes in SY-PPV balance out to give a relative quantum yield $\phi_{\mathrm{f}}$, which is fairly temperature independent.

In $\mathrm{BEH}-\mathrm{PPV}$, the decrease of $k_{\mathrm{nr}}(300-150 \mathrm{~K})$ results from the decrease in internal vibrational energy redistribution pathways due to the changes in inter- and intrachain packing. This is consistent with the decreased electron-vibrational coupling at lower temperatures, evidenced by the changing peak ratio between $S_{0,0}$ and $S_{0,1}$ (Fig. 2d). At lower temperatures ( $<150 \mathrm{~K}$ ), $k_{\mathrm{nr}}$ increases. Previous work, including vibrational spectroscopy, has shown that the electronic-vibrational coupling in BEH-PPV is temperature dependent, with even additional vibronic peaks being resolved at temperatures lower than what we have studied here. ${ }^{26}$ Such an effect was also observed in MEH-PPV. ${ }^{41}$ The increase in $k_{\mathrm{nr}}$ at lower temperatures is therefore attributed to increased energy transfer processes from shorter to longer conformational subunits as well as a possible change in the electron-vibrational coupling. The increased excited state lifetime at lower temperatures in BEH-PPV mainly originates from the decrease in exciton quenching due to slower diffusion toward traps (decrease in $k_{\text {diff }}$ ), since $k_{\text {diff }}$ is an order of magnitude larger as compared to $k_{\mathrm{nr}}$. The reduction of these non-radiative processes at lower temperatures, dominated by a reduced $k_{\text {diff }}$, then give rise an increase in the relative quantum yield $\phi_{\mathrm{f}}$ as observed in BEH-PPV.

The electronic and optical properties of conductive polymer films may also be influenced by thermal treatment at or around the glass transition temperature, $T_{\mathrm{g} \cdot}{ }^{42,43}$ For PPV based polymers, $T_{\mathrm{g}}$ values reported in literature are above room temperature. ${ }^{44,45}$ We therefore do not expect this to influence our results, where the highest temperature measured is $298 \mathrm{~K}$.

\section{Downhill migration depends on energetic disorder}

We found further, that at higher temperatures, the less disordered polymer BEH-PPV exhibits less temperature dependence on $D$ and $k_{\text {diff. }}$ This is consistent with the low energetic disorder in this system. Since the quasi-equilibrium state is due to the narrow $\sigma$ close to the center of the DOS, fast energy transfer between the conformational subunits proceeds, represented by a high exciton diffusion coefficient at room temperature $\left(2.9 \times 10^{-3} \mathrm{~cm}^{2} \mathrm{~s}^{-1}\right)$. While cooling down to the characteristic temperature $(<120 \mathrm{~K})$, the exciton diffusion coefficient decreases slightly to $2.0 \times 10^{-3} \mathrm{~cm}^{2} \mathrm{~s}^{-1}$, which is 
consistent with the decrease in $k_{\text {diff. }}$ Below $120 \mathrm{~K}$, where thermally activated hopping is absent, the exciton diffusion coefficient drops down to $8.3 \times 10^{-4} \mathrm{~cm}^{2} \mathrm{~s}^{-1}$ and $k_{\text {diff }}$ to $1.79 \times 10^{9} \mathrm{~s}^{-1}$, respectively. Due to the narrow energy spread in BEH-PPV, with decreasing temperature there are still enough possible energy-sites for the excitons to jump while the equilibrium-state shifts down into the Gaussian tail. Thus, the exciton diffusion coefficient of BEH-PPV at $120 \mathrm{~K}$ is still one order of magnitude higher than the one of SY-PPV at room temperature $\left(3.1 \times 10^{-4} \mathrm{~cm}^{2} \mathrm{~s}^{-1}\right)$, while $k_{\text {diff }}$ is two orders of magnitude higher (see also Tables 3 and 4 ).

In contrast to the exciton diffusion coefficient, the exciton diffusion length $L_{\mathrm{D}}$ of BEH-PPV first increases while cooling to $180 \mathrm{~K}$. This increase from $7.7 \mathrm{~nm}$ to $9.6 \mathrm{~nm}$ is a consequence of the increased exciton lifetime resulting from less competitive non-radiative decay rates with lowering temperature (see eqn (5)). Then, in between $180 \mathrm{~K}$ and $120 \mathrm{~K}$ the $L_{\mathrm{D}}$ of BEH-PPV slightly decreases. The previously noted change in electronicvibrational coupling could affect the disorder parameter $\sigma$ and therefore also the temperature dependence of $L_{\mathrm{D}}$. Finally, at a characteristic temperature of $100 \mathrm{~K}$, the exciton diffusion is fully determined by downhill migration and the exciton diffusion length drops off from $8.9 \mathrm{~nm}$ to $5.2 \mathrm{~nm}$.

Since the thermal equilibrium state $-\sigma^{2} / k T$ shifts with increasing disorder strength $\sigma$ deeper into the tail states of the Gaussian DOS, the probability to find a nearby energy-site to which a jump is probable decreases noticeably in the case of a more disordered polymer. ${ }^{37}$ Therefore, in SY-PPV the exciton migration toward quenching defects is much slower at room temperature, leading to an order of magnitude longer exciton lifetime (1.9 ns) compared to BEH-PPV (200 ps). ${ }^{14}$ At room temperature, the slow exciton diffusion in the disordered SY-PPV is represented by a low exciton diffusion coefficient of $3.1 \times 10^{-4} \mathrm{~cm}^{2} \mathrm{~s}^{-1}$ and the two orders of magnitude lower transfer rate $k_{\text {diff }}$ (Tables 3 and 4 ) compared to BEH-PPV. The decrease in $D$ and $L_{\mathrm{D}}$, while cooling to $180 \mathrm{~K}$ (Fig. 3), is a direct consequence of the down-shift of the equilibrium level into the tail of the Gaussian DOS, where the energy difference between occupied and neighboring sites increases, leading to an even lower $D\left(8.0 \times 10^{-5} \mathrm{~cm}^{2} \mathrm{~s}^{-1}\right)$ and $k_{\text {diff. }}$ This lower $D$ along with a nearly constant exciton lifetime results in a decrease of $L_{\mathrm{D}}$ from $7.7 \mathrm{~nm}$ to $4.2 \mathrm{~nm}$ in the temperature range of $290-180 \mathrm{~K}$. Below the characteristic temperature $(180 \mathrm{~K})$, where thermally activated hopping is switched off, the exciton diffusion becomes fully determined by downhill migration, represented by temperatureindependent $D\left(\sim 6.8 \times 10^{-5} \mathrm{~cm}^{2} \mathrm{~s}^{-1}\right)$ and $L_{\mathrm{D}}(\sim 3.9 \mathrm{~nm})$.

The better ordered BEH-PPV exhibits PL characteristics that are temperature sensitive and are dominated by the exciton diffusionlimited quenching model. The increase in $L_{\mathrm{D}}$ of BEH-PPV with lowering temperature is governed by the increase in $\tau_{\mathrm{f}}$, while in the case of SY-PPV temperature-dependence of $L_{\mathrm{D}}$ is dominated by the temperature-dependence of $D$. Our findings demonstrate that the temperature at which thermally activated hopping diminishes scales with the energetic disorder. Better ordered PPV derivatives correlate with lower transition temperatures between thermally activated hopping and downhill migration.

\section{Origin of PLED efficiency enhancement in BEH-PPV at low temperature}

PLEDs based on these materials demonstrate that BEH-PPV exhibits temperature dependent efficiencies while SY-PPV does not. The current efficiency (CE) in PLEDs is defined as light output divided by current (exciton/charge carrier), and describes the relation between the number of emitted photons divided by the number of charges that passed the device in the same time interval. Since the charge current and the recombination prefactors of the radiative Langevin and nonradiative ShockleyRead-Hall recombination scale linearly with mobility, upon dividing these rates for current and light output, the mobility drops out and the efficiency is expected to be mobility independent. ${ }^{6,40,46}$ In a space-charge limited device such as PLEDs, the number of charge carriers is mainly determined by the applied voltage and not by the temperature. Therefore, the current efficiency in PLEDs is reported to be temperatureindependent. ${ }^{17}$ Previous work on the temperature-dependent characteristics of PLEDs have focused on the role of the chargeinjection barrier with temperature. ${ }^{18,47}$

However, the PLED efficiency also depends on the decay dynamics of a formed exciton, ${ }^{17,48}$ which is described by the PL efficiency. The temperature-independence of the CE in the high disordered polymer SY-PPV PLED (Fig. 4a) is explained as follows: after formation of the exciton, it subsequently diffuses along the conjugated chain segments until it reaches a radiative or non-radiative recombination center and decays either radiatively or non-radiatively. The increased population of non-luminescent conformational subunits present in the SY-PPV copolymer compensates for the decrease in exciton diffusion toward non-radiative quenching-sites with lowering temperature. Therefore, the current efficiency, which directly correlates with the PL efficiency $\phi_{\mathrm{f}}$, shows no temperaturedependence in the SY-PPV based PLED.

In contrast, the increased current efficiency in the less disordered BEH-PPV PLED in the temperature-range of 295-215 K (Fig. 4b) is a direct consequence of enhanced $\phi_{\mathrm{f}}$, resulting from less competitive non-radiative diffusion-dominated decay processes with lowering temperature. We note that the temperaturedependence of the current efficiency in BEH-PPV is stronger than that of the relative quantum yield $\phi_{\mathrm{f}}$. This can be attributed to the difference in the relative intensity of peaks in the EL spectra compared to the PL spectra due to interference in the optical microcavity of the EL device. This will influence the device efficiency and the rate of spontaneous emission. ${ }^{32}$ In the optical microcavity, the BEH-PPV EL spectra still demonstrate decreased electron-vibrational coupling with decreasing temperature. This is evidenced by the increasing ratio between the $S_{0,0}$ and $S_{0,1}$ peaks (Fig. $4 \mathrm{~d}$ ), and is consistent with the decrease in $k_{\mathrm{nr}}$ noted in this temperature range in the PL measurements.

In addition to the different responses to temperature, the SY-PPV PLED exhibits an order of magnitude higher CE than the BEH-PPV PLED. The lower CE in BEH-PPV is a direct result of the faster exciton diffusion ( $\left.k_{\text {diff }}\right)$ towards non-radiative traps, 
and is consistent with the photoluminescence measurements. As mentioned above, Fig. 5 demonstrates that, at room temperature, the exciton quenching at defects is $90 \%$ in BEH-PPV and $10 \%$ in SY-PPV. As a result, while the same amount of excitons are formed in the two materials for a given current, the amount of photons generated differs by an order of magnitude due to the difference in the kinetically competitive quenching at non-radiative traps. This results in a higher CE in the more energetically disordered SY-PPV.

\section{Conclusions}

We have shown that the temperature-dependent excited state lifetime, relative quantum yield, and exciton diffusion characteristics, i.e. exciton diffusion coefficient and exciton diffusion length, depend on the energetic disorder of the conjugated polymer system. The less disordered polymer BEH-PPV shows a decrease in non-radiative decay due to both freezing out vibrations (influences $k_{\mathrm{nr}}$ ) with decreasing temperature and less exciton quenching due to slowing down the diffusion toward traps (decrease in $k_{\text {diff }}$ ), leading to increase in exciton lifetime and relative quantum yield. Since the CE of a PLED is directly correlated to the PL efficiency, we found in that the PLED based on the better ordered polymer showed enhanced CE at low temperatures due to reduced quenching at defect centers. Polymers with higher energetic disorder, as in SY-PPV, show different temperature-dependence of the exciton diffusion properties. With lowering temperature the exciton lifetime slightly increases, while the relative quantum yield decreases, due to different conformational subunits and increased competitions from radiative and non-radiative sites. As a consequence, PLEDs based on strongly disordered polymers show nearly temperature independent conversion efficiencies and PLQYs, respectively.

\section{Conflicts of interest}

There are no conflicts to declare.

\section{Acknowledgements}

The work of I. R. is part of the research program of the Dutch Polymer Institute (project \#763). The authors are grateful to Gert-Jan A. H. Wetzelaer and Jasper Michels for helpful discussions and to Dominik Gehrig, Simon A. Bretschneider, Frank Keller, and Hans-Jürgen Guttmann for their technical support. Open Access funding provided by the Max Planck Society.

\section{Notes and references}

1 H. Bässler, Phys. Status Solidi, 1993, 175, 15-56.

2 A. Köhler and H. Bässler, Electronic Processes in Organic Semiconductors, Wiley-VCH Verlag GmbH \& Co. KGaA, Weinheim, Germany, 2015.

3 T. Tiedje and A. Rose, Solid State Commun., 1981, 37, 49-52. 4 D. D. C. Bradley, Synth. Met., 1993, 54, 401-415.
5 J. H. Burroughes, D. D. C. Bradley, A. R. Brown, R. N. Marks, K. Mackay, R. H. Friend, P. L. Burns and A. B. Holmes, Nature, 1990, 347, 539-541.

6 M. Kuik, G.-J. A. H. Wetzelaer, H. T. Nicolai, N. I. Craciun, D. M. De Leeuw and P. W. M. Blom, Adv. Mater., 2014, 26, 512-531.

7 B. Movaghar, M. Grünewald, B. Ries, H. Bassler and D. Würtz, Phys. Rev. B: Condens. Matter Mater. Phys., 1986, 33, 5545-5554.

8 M. Grünewald, B. Pohlmann, B. Movaghar and D. Würtz, Philos. Mag. B, 1984, 49, 341-356.

9 O. V. Mikhnenko, F. Cordella, A. B. Sieval, J. C. Hummelen, P. W. M. Blom and M. A. Loi, J. Phys. Chem. B, 2008, 112, 11601-11604.

10 S. Athanasopoulos, E. Hennebicq, D. Beljonne and A. B. Walker, J. Phys. Chem. C, 2008, 112, 11532-11538.

11 T. Q. Nguyen, V. Doan and B. J. Schwartz, J. Chem. Phys., 1999, 110, 4068-4078.

12 J. R. Lakowicz, Principles of Fluorescence Spectroscopy, Kluwer, Dordrecht, 1999.

13 O. V. Mikhnenko, P. W. M. Blom and T.-Q. Nguyen, Energy Environ. Sci., 2015, 8, 1867-1888.

14 I. Rörich, O. V. Mikhnenko, D. Gehrig, P. W. M. Blom and N. I. Crăciun, J. Phys. Chem. B, 2017, 121, 1405-1412.

15 O. V. Mikhnenko, M. Kuik, J. Lin, N. van der Kaap, T.-Q. Nguyen and P. W. M. Blom, Adv. Mater., 2014, 26, 1912-1917.

16 D. E. Markov, C. Tanase, P. W. M. Blom and J. Wildeman, Phys. Rev. B: Condens. Matter Mater. Phys., 2005, 72, 045217.

17 P. W. M. Blom, M. J. M. De Jong and S. Breedijk, Appl. Phys. Lett., 1997, 71, 930-932.

18 S. J. Martin, J. M. Lupton, I. D. W. Samuel and A. B. Walker, J. Phys.: Condens. Matter, 2002, 14, 9925-9933.

19 I. H. M. van Stokkum, D. S. Larsen and R. van Grondelle, Biochim. Biophys. Acta, Bioenerg., 2004, 1657, 82-104.

20 K. M. Mullen and I. H. M. van Stokkum, J. Stat. Softw., 2007, 18, 1-46.

21 J. J. Snellenburg, S. P. Laptenok, R. Seger, K. M. Mullen and I. H. M. van Stokkum, J. Stat. Softw., 2012, 49, 1-22.

22 O. V. Mikhnenko, H. Azimi, M. Scharber, M. Morana, P. W. M. Blom and M. A. Loi, Energy Environ. Sci., 2012, 5, 6960.

23 O. V. Mikhnenko, J. Lin, Y. Shu, J. E. Anthony, P. W. M. Blom, T.-Q. Nguyen and M. A. Loi, Phys. Chem. Chem. Phys., 2012, 14, 14196-14201.

24 O. V. Mikhnenko, Software for Monte Carlo simulations, http://mikhnenko.com/eDiffusion/.

25 E. W. Snedden, L. A. Cury, K. N. Bourdakos and A. P. Monkman, Chem. Phys. Lett., 2010, 490, 76-79.

26 F. A. C. Oliveira, L. A. Cury, A. Righi, R. L. Moreira, P. S. S. Guimarães, F. M. Matinaga, M. A. Pimenta and R. A. Nogueira, J. Chem. Phys., 2003, 119, 9777-9782.

27 S. Gambino, A. K. Bansal and I. D. W. Samuel, Org. Electron., 2013, 14, 1980-1987.

28 A. Bruno, L. X. Reynolds, C. Dyer-Smith, J. Nelson and S. A. Haque, J. Phys. Chem. C, 2013, 117, 19832-19838.

29 A. Haugeneder, M. Neges, C. Kallinger, W. Spirkl, U. Lemmer, J. Feldmann, U. Scherf, E. Harth, A. Gügel 
and K. Müllen, Phys. Rev. B: Condens. Matter Mater. Phys, 1999, 59, 15346-15351.

30 S. R. Scully and M. D. McGehee, J. Appl. Phys., 2006, 100, 034907.

31 D. Abbaszadeh, A. Kunz, G.-J. A. H. Wetzelaer, J. J. Michels, N. I. Crăciun, K. Koynov, I. Lieberwirth and P. W. M. Blom, Nat. Mater., 2016, 15, 628-633.

32 M. Furno, R. Meerheim, S. Hofmann, B. Lüssem and K. Leo, Phys. Rev. B: Condens. Matter Mater. Phys., 2012, 85, 115205.

33 R. H. Friend, R. W. Gymer, A. B. Holmes, J. H. Burroughes, R. N. Marks, C. Taliani, D. D. C. Bradley, D. A. Dos Santos, J. L. Brédas, M. Lögdlund and W. R. Salaneck, Nature, 1999, 397, 121-128.

34 B. Mollay, U. Lemmer, R. Kersting, R. F. Mahrt, H. Kurz, H. F. Kauffmann and H. Bässler, Phys. Rev. B: Condens. Matter Mater. Phys., 1994, 50, 10769-10779.

35 R. Kersting, U. Lemmer, R. F. Mahrt, K. Leo, H. Kurz, H. Bässler and E. O. Göbel, Phys. Rev. Lett., 1993, 70, 3820-3823.

36 M. Anni, M. E. Caruso, S. Lattante and R. Cingolani, J. Chem. Phys., 2006, 124, 134707.

37 S. Athanasopoulos, E. V. Emelianova, A. B. Walker and D. Beljonne, Phys. Rev. B: Condens. Matter Mater. Phys, 2009, 80, 195209.

38 S. T. Hoffmann, H. Bässler, J. M. Koenen, M. Forster, U. Scherf, E. Scheler, P. Strohriegl and A. Köhler, Phys. Rev. B: Condens. Matter Mater. Phys., 2010, 81, 115103.
39 M. R. Andersson, G. Yu and A. J. Heeger, Synth. Met., 1997, 85, 1275-1276.

40 Q. Niu, G.-J. A. H. Wetzelaer, P. W. M. Blom and N. I. Crăciun, Adv. Electron. Mater., 2016, 2, 1600103.

41 M. A. T. Da Silva, I. F. L. Dias, J. L. Duarte, E. Laureto, I. Silvestre, L. A. Cury and P. S. S. Guimarães, J. Chem. Phys., 2008, 128, 094902.

42 J. Liu, T.-F. Guo and Y. Yang, J. Appl. Phys., 2002, 91, 1595-1600.

43 D. Kajiya, T. Koganezawa and K. Saitow, AIP Adv., 2015, 5, 127130.

44 O. Schäfer, A. Greiner, J. Pommerehne, W. Guss, H. Vestweber, H. Y. Tak, H. Bässler, C. Schmidt, G. Lüssem, B. Schartel, V. Stümpflen, J. H. Wendorff, S. Spiegel, C. Möller and H. W. Spiess, Synth. Met., 1996, 82, 1-9.

45 R. I. Venkatanarayanan, S. Krishnan, A. Sreeram, P. A. Yuya, N. G. Patel, A. Tandia and J. B. McLaughlin, Macromol. Theory Simul., 2016, 25, 238-253.

46 M. Kuik, L. J. A. Koster, G.-J. A. H. Wetzelaer and P. W. M. Blom, Phys. Rev. Lett., 2011, 107, 256805.

47 J. M. Lupton and I. D. W. Samuel, Synth. Met., 2000, 111-112, 381-384.

48 P. W. M. Blom and M. J. M. De Jong, IEEE J. Sel. Top. Quantum Electron., 1998, 4, 105-112. 